\title{
Finding Earth clones with SIM: the most promising near-term technique to detect, find masses for, and determine three-dimensional orbits of nearby habitable planets
}

Michael Shao, Stephen C. Unwin, Charles Beichman, Joseph Catanzarite, Stephen J. Edberg, et al.

Michael Shao, Stephen C. Unwin, Charles Beichman, Joseph Catanzarite, Stephen J. Edberg, James C. Marr, Geoffrey Marcy, "Finding Earth clones with SIM: the most promising near-term technique to detect, find masses for, and determine three-dimensional orbits of nearby habitable planets," Proc. SPIE 6693, Techniques and Instrumentation for Detection of Exoplanets III, 66930C (19 September 2007); doi: 10.1117/12.734671

SPIE Event: Optical Engineering + Applications, 2007, San Diego, California, United States 


\title{
Finding Earth Clones with SIM
}

\section{The Most Promising Near-Term Technique to Detect, Find Masses for, and Determine Three-dimensional Orbits of Nearby Habitable Planets}

\author{
Michael Shao* ${ }^{1}$, Stephen C. Unwin ${ }^{1}$, Charles Beichman ${ }^{2}$, Joseph Catanzarite ${ }^{1}$, Stephen J. Edberg ${ }^{1}$, \\ James C. Marr-IV ${ }^{1}$, Geoffrey Marcy ${ }^{3}$ \\ ${ }^{1}$ Jet Propulsion Laboratory, California Institute of Technology, 4800 Oak Grove Drive, Pasadena, CA 91109 \\ ${ }^{2}$ Michelson Science Center, California Institute of Technology, 1200 E. California Blvd., Pasadena, CA 91125 \\ ${ }^{3}$ University of California, Berkeley, 417 Campbell Hall, Berkeley, CA 94720
}

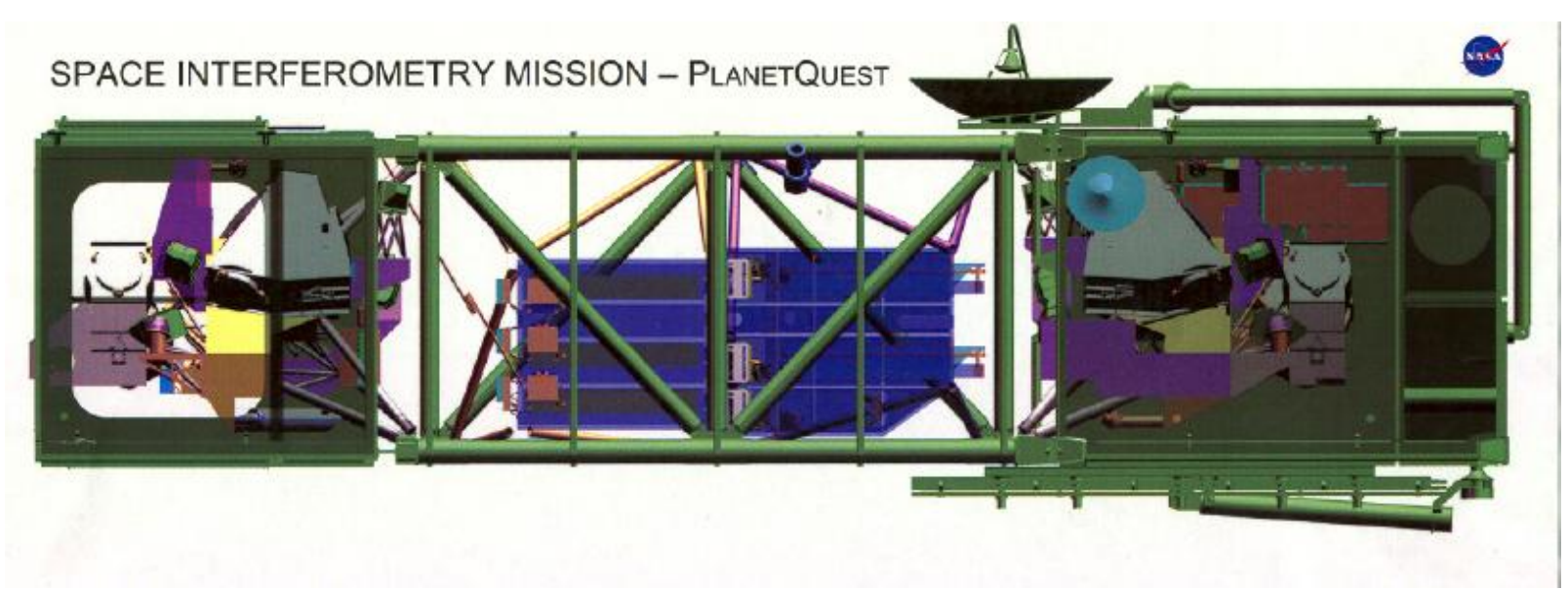

\begin{abstract}
SIM is a space astrometric interferometer capable of better than one-microarcsecond ( $\mu$ as) single measurement accuracy, providing the capability to detect stellar "wobble" resulting from planets in orbit around nearby stars. While a search for exoplanets can be optimized in a variety of ways, a SIM five-year search optimized to detect Earth analogs $(0.3$ to 10 Earth masses) in the middle of the habitable zone (HZ) of nearby stars would yield the masses, without $\mathrm{M}^{*} \sin (\mathrm{i})$ ambiguity, and three-dimensional orbital parameters for planets around $\sim 70$ stars, including those in the $\mathrm{HZ}$ and further away from those same stars. With $>200$ known planets outside our solar system, astrophysical theorists have built numerical models of planet formation that match the distribution of Jovian planets discovered to date and those models predict that the number of terrestrial planets $\left(<10 \mathrm{M}_{\oplus}\right)$ would far exceed the number of more massive Jovian planets. Even so, not every star will have an Earth analog in the middle of its HZ. This paper describes the relationship between SIM and other planet detection methods, the SIM planet observing program, expected results, and the state of technical readiness for the SIM mission.
\end{abstract}

*michael.shao@jpl.nasa.gov; phone 818-354-7834; fax 818-393-2412; jpl.nasa.gov

\footnotetext{
Techniques and Instrumentation for Detection of Exoplanets III, edited by Daniel R. Coulter Proc. of SPIE Vol. 6693, 66930C, (2007) · 0277-786X/07/\$18 - doi: 10.1117/12.734671
} 


\section{INTRODUCTION}

Detection and characterization of one-Earth-mass $\left(1 \mathrm{M}_{\oplus}\right)$ planets in the habitable zone (HZ) of nearby stars is the ultimate objective of the NASA Navigator Program. One method of detecting these planets is ultra-precise optical astrometry with a Michelson stellar interferometer to track the motion of the star/planet-system about its barycenter over a period of time yielding mass, without $\mathrm{M}^{*} \sin (\mathrm{i})$ ambiguity, and complete orbit characterization for all planets within the range of detection (lower bound limited by astrometric precision and stellar distance; upper bound limited by mission duration and stellar distance). Detailed simulations (Catanzarite 2006, Shao 2007) have shown that one microarcsecond $\left(1 \mu\right.$ as) relative astrometry provides the required capability to detect $1 \mathrm{M}_{\oplus}$ planets in the $\mathrm{HZ}$ of 110 of our closest stars over a ten year mission (or $\sim 65$ over a five year mission) using about $37 \%$ of mission time. With SIM's current best estimate (CBE) performance of $0.6 \mu$ as, SIM could search 129 nearby stars in five years for $1 \mathrm{M}_{\oplus}$ planets in the $\mathrm{HZ}$ and for both smaller and larger planets further away from the star than the HZ.

To date, SIM remains the only program with the capability of detecting and confirming rocky planets in the habitable zones of nearby solar-type main sequence stars (types F, G, \& K). Moreover, SIM targets are the only stars for which follow-up by other techniques is feasible, such as space-based spectroscopy, ground-based interferometry, and the terrestrial planet finders (TPF).

\section{SIM BACKGROUND}

Optical and infrared interferometry have been well developed for ground applications such as NPOI, KI, VLTI, CHARA, etc., yet these facilities suffer limitations due to coherence size, angle, and time imposed by the Earth's atmosphere. The National Research Council (NRC) recognized in 1990 that a space-based Astrometric Interferometry Mission (AIM) capable of $30 \mu$ as performance "would have a great impact on many branches of astronomy," including the ability to detect planets as far away as 500 parsec (Bahcall 1991). This mission concept was reaffirmed in 2000 in the Astronomy and Astrophysics in the New Millenium (AANM) report (McKee/Taylor 2001) and again by the Committee on Astronomy and Astrophysics (CAA) in 2002 (McElroy/Huchra 2002) with required performance revised to $10 \mu$ as (goal $4 \mu \mathrm{as}$ ) and the added requirement for achieving $3 \mu$ as (goal $1 \mu$ as) narrow-angle precision for the purpose of detection of terrestrial (rocky) planets around nearby stars. All three of these reports also recognized the benefit of AIM/SIM technology demonstrations to future space-based very-long baseline optical and infrared interferometers that might be capable of direct detection and eventually direct rotational synthesis imaging of planets around these nearby stars.

The challenge that NASA accepted was to convert ground-demonstrated concepts into space-capable hardware and software with sufficient reliability to enable an unattended five to ten year space-based mission. A program was undertaken at JPL to identify and develop the necessary technologies to enable space-based interferometry missions. This technology development was completed in 2005 demonstrating technology readiness level six (TRL6) with final review and signoff by NASA HQ in March 2006. This program demonstrated the ability to achieve $0.6 \mu$ as narrowangle performance and $2.4 \mu$ as wide-angle performance, $40 \%$ better than the goal performance in both cases.

\section{ASTROMETRIC DETECTION OF PLANETS}

SIM is an optical Michelson interferometer with a nine meter baseline. Fringe detection with a CCD allows measurement of wavefront delays, leading to an angular precision of $0.6 \mu$ as in a single measurement and a systematic error floor below $0.1 \mu$ as (for longer integration times) as verified with the SIM optical testbed at JPL. For planet detection, SIM measures angular positions of stars to a single measurement precision of $0.6 \mu$ as for stars as faint as $7^{\text {th }}$ mag (in V) relative to $10^{\text {th }}$ mag reference stars within $\sim 1$ degree of the target star. About 200 one-dimensional visits of $\sim 20$ minutes each spread over five years for each star are used to determine stellar wobble resulting from the motion of the star and planets around their barycenter.

SIM's planet detection thresholds follow directly. As a benchmark, an Earth-mass planet orbiting 1 AU from a solar mass star at $6 \mathrm{pc}$ induces an angular wobble with a radius of $0.5 \mu$ as. SIM can detect this since its demonstrated systematic error floor is below $0.1 \mu$ as With $\mathrm{N}$ measurements spanning a few orbits, the detection improves as the square root of $\mathrm{N}$ because the signal's periodicity is coherent (inconsistent with random or systematic errors). 
While radial velocity (RV) detection is more sensitive to close-in orbits, astrometry is more sensitive for more distant orbits (Fig. 1). Around stars more luminous than the Sun, an Earth in the habitable zone (HZ) can be detected by SIM in a large volume of space in the solar neighborhood.

\section{Exoplanet Discovery Space}

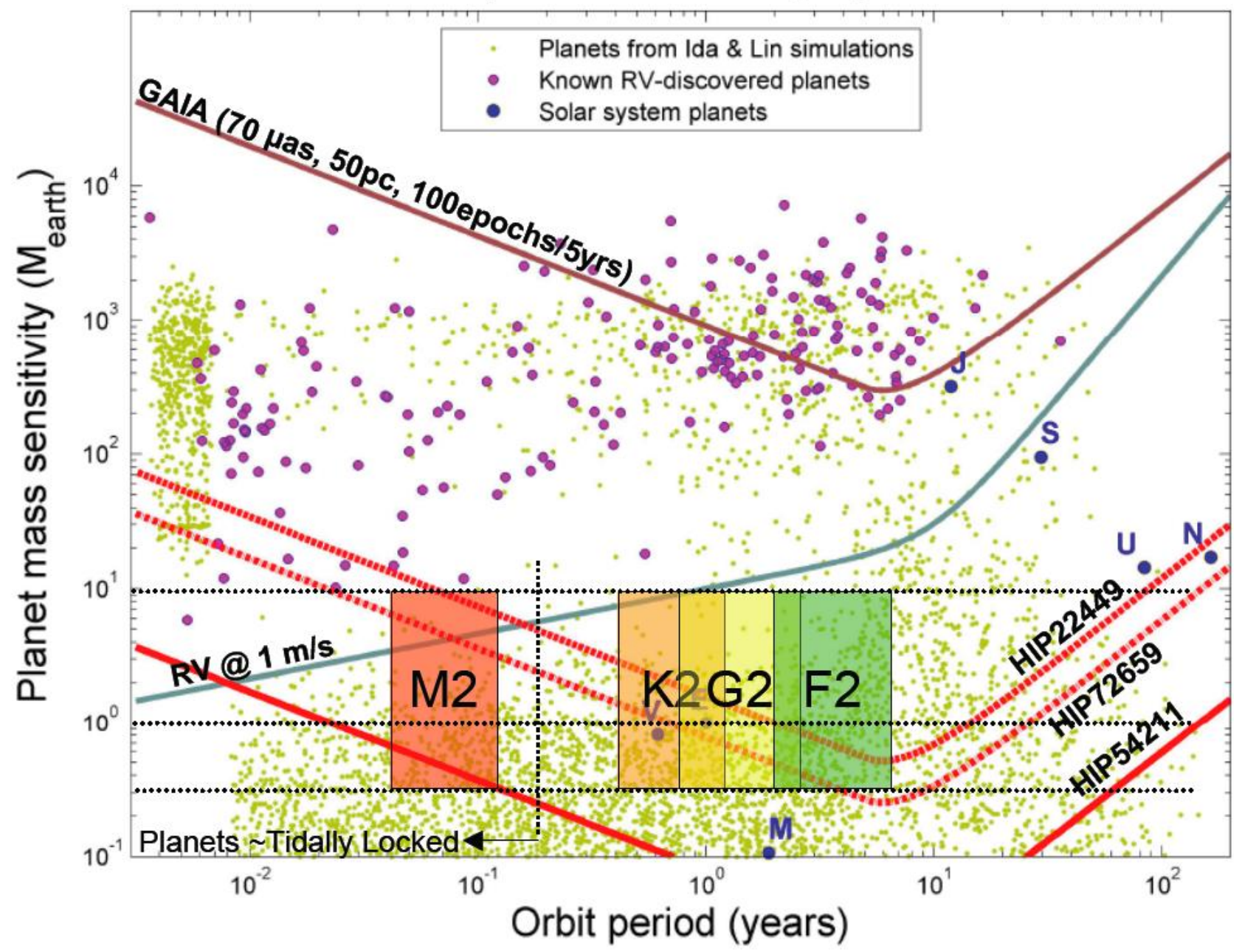

Fig. 1: SIM is the only mission that explores almost the entire range of masses and orbit periods where terrestrial planets form and evolve. The solid red curve is for the most favorable of 129 candidate stars (HIP52411), while the least favorable star (HIP22449) is about an order of magnitude above this line. (The discovery space for each star lies above the line shown). Yellow dots represent planet distributions from the simulations of Ida \& Lin (2004ab), and purple dots show the known RV detections. The habitable zone for M2 through F2 stars with rocky planets in the 0.3 to 1.0 Earth-mass range are shown for reference. (Unwin 2007)

With $0.6 \mu$ as single measurement accuracy, SIM could detect one-Earth-mass planets orbiting at mid-habitable zone around every one of the most favorable 129 stars in the solar neighborhood using $40 \%$ of total mission time (see Table 1). Limiting the depth of the habitable-zone mass search to larger than $1 \mathrm{M}_{\oplus}$ planets would enable more stars to be searched as shown in Table 1 for $2 \mathrm{M}_{\oplus}$ and $3 \mathrm{M}_{\oplus}$ search depth. Table 1 also shows estimates of planets of various masses that might be discovered based upon models of planetary formation. It is important to recognize that these are indeed just estimates and the purpose of actually making the measurements is to test the models. As can be seen in Fig 1 , a survey designed to detect $1 \mathrm{M}_{\oplus}$ planets in the mid-habitable zone will also detect planets of lower mass further out from the star as well as more massive planets limited only by the duration of the mission. 
Choice of the depth of search can be made pre-launch depending upon whether the results of the NASA Kepler mission reveal that rocky planets are common (as current models suggest) or rare. Should they be rare, the best strategy may be to do a shallower (down to a larger minimum mass) search of many more stars rather than a deeper (down to $1 \mathrm{M}_{\oplus}$ ) search.

Table 1. SIM Habitable Planet Survey

\begin{tabular}{|l|l|l|l|}
\hline $\begin{array}{l}\text { Mass sensitivity at mid } \\
\text { HZ } 0.6 \mu \text { as }\end{array}$ & $1 \mathrm{M}_{\oplus}$ & $2 \mathrm{M}_{\oplus}$ & $3 \mathrm{M}_{\oplus}$ \\
\hline \# of target stars searched & 129 & 300 & 488 \\
\hline $\begin{array}{l}\text { \# Terrestrial planets (0.3 } \\
-10 \mathrm{M}_{\oplus} \text { ) expected (from } \\
\text { models) }\end{array}$ & 44 & 68 & 85 \\
\hline $\begin{array}{l}\text { \# Terrestrial planets in } \\
\text { the HZ expected (from } \\
\text { models) }\end{array}$ & 10 & 16 & 18 \\
\hline $\begin{array}{l}\text { Total number of planets } \\
\text { expected (from models) }\end{array}$ & 73 & 129 & 181 \\
\hline
\end{tabular}

Many of the terrestrial planets found by SIM, because they are around nearby stars, can be followed up by ambitious observations made with telescopes on the ground and in space. Moreover, SIM's direct measurement of the astrometric wobble yields the mass of the planet unambiguously (no $\mathrm{M}^{*} \sin$ $i$ ambiguity). Equally important, the full three-dimensional orbit for all planets found are derived from SIM astrometry, yielding the orbital eccentricity of single planets and the coplanarity of multiple planets in a system. Co-planarity is often assumed, but without any observational evidence; SIM measures it, unequivocally. Thus, SIM offers the unique ability, among proven techniques, to both: (1) detect the habitable planets around the nearest stars and (2) to give the masses, eccentricities, and co-planarities that are so important for testing planet-formation theory.

With the technology fully tested and vetted, SIM is ready to become the first mission to identify and analyze rocky planets orbiting stars in the solar neighborhood. Such detections would motivate numerous follow-up observations especially from space, launching the new field of habitable planet science.

\section{SIM EXOPLANET SEARCH PROGRAMS}

The known exoplanets have orbital period and mass distributions that are roughly uniform in log-period and log-mass, respectively. Analysis of 200 known planets yields power laws for the distribution in mass and period of dN/dM $\sim \mathrm{M}^{-1}$ and $\mathrm{dN} / \mathrm{dP} \sim \mathrm{P}^{-1}$ (Cumming et al. 2007).

SIM detects the presence of a planet by measuring the periodic reflex motion of the star as the planet orbits the star. Ideally, the motion is observed for at least one orbital period. If the orbital period is longer than the duration of observations, planet detectability is degraded due to confusion with proper motion. After a series of observations have been made, we may compute the periodogram power with the detection threshold set so that the false alarm probability is less than $1 \%$.

The SIM exoplanet program consists of three major components:

(a) A habitable planet survey focusing on the nearest stars. These are planets between 0.3 and 10 times the mass of the Earth, orbiting in the habitable zone, which ranges from 0.7 to $1.4 \mathrm{AU}$ from the star, scaled appropriately for stellar luminosity.

(b) A broad planet survey of $\sim 2100$ stars. The broad survey at $\sim 4$ times lower astrometric accuracy but with $\sim 20$ times as many targets will be able to find terrestrial mass planets in five year orbits around low mass stars.

(c) A young star planet survey focusing on Jupiter/Saturn mass planets orbiting very young stars, some of which are young enough that the planet formation and migration processes are still underway.

Subheading 6 describes how SIM is synergistic with other planet detection techniques.

\section{4a. SIM Habitable Planet Survey}

The SIM habitable planet survey can be optimized by considering fiducial A,F,G,K stars in the solar neighborhood. One may calculate the orbital distance to the $\mathrm{HZ}$ for each star and rank the stars in terms of astrometric detectability. For the habitable planet survey, each target is allocated just enough observing time to detect a $1 \mathrm{M}_{\oplus}$ planet at the middle of its habitable zone. SIM can conduct a HZ search of 129 stars with sensitivity down to $1 \mathrm{M}_{\oplus}$. The amount of observing time 
needed increases as $1 /$ (planet mass) $^{2}$ so that a larger number of stars can be searched by relaxing the mass sensitivity to 2 or $3 \mathrm{M}_{\oplus}$ (Table-1).

The science return can be estimated based on the expected distribution of planets in mass and orbital parameter space. From the 200 known exoplanets spanning the range from $7 \mathrm{M}_{\oplus}$ to 10 Jupiter masses and orbital radii from 0.05 AU to 3 $\mathrm{AU}$, the probability distributions of mass and orbit radius can be extrapolated into the long-period and low-mass regime. Recent theoretical models of rocky planet formation (Ida \& Lin 2004ab, 2005 and Benz et al. 2006) predict that there are many more $<10 \mathrm{M}_{\oplus}$ planets than Jupiters. Benz expects a $\sim 5$ fold increase in terrestrial planets over a simple extrapolation of the power-law model for higher mass planets.

Accordingly, we adopt for our model of the probability distribution of planets the power laws derived from the known RV-detected planets by Cumming et al. (2007), but bump up the density of planets below $10 \mathrm{M}_{\oplus}$ by a factor of five. This model predicts that $\sim 73 \%$ of stars have a terrestrial planet, $\sim 10 \%$ of stars have terrestrial planets in the $\mathrm{HZ}$ and $\sim 1 \%$ of stars will have Earth 'clones' $\left(0.8 \sim 1.2 \mathrm{M}_{\oplus}, 0.7 \sim 1.4 \mathrm{AU}\right)$. Using this model one may estimate how many terrestrial planets SIM would find, as indicated in Table 1 . The model also predicts that $\sim 22 \%$ of stars have planets more massive than $10 \mathrm{M}_{\oplus}$.

\section{4b. SIM Broad Planet Survey}

The SIM broad planet survey is a comprehensive search for planets, down to terrestrial mass, around $\sim 2100$ stars. These target stars cover essentially the full range of stellar properties and the hundreds of planets found will form the largest sample of nearby planets with precisely known orbits and masses. This unique sample will set the target lists for future exoplanet imaging and spectroscopic missions.

The survey, comprising just $4 \%$ of SIM mission time, has been designed to detect terrestrial-mass and larger planets with orbital periods of several years. To enable coverage of a large number of targets, the survey has a somewhat lower astrometric precision than the $\mathrm{HZ}$ planet-search portion of the SIM mission.

This survey has a nominal single-measurement astrometric precision of $4 \mu$ as, which allows unambiguous detection of 2Earth-mass planets in five-year orbital periods around solar-mass stars at $10 \mathrm{pc}$ distance ( $1 \%$ false-alarm probability). The survey is sensitive to sub-Earth-mass planets around M-dwarfs and terrestrial-mass planets around all but the most massive stars in the sample. Multiple-planet systems will be readily identifiable. Precise three-dimensional orbital solutions will provide mass, orbit semimajor axis, inclination, and eccentricity for each detected planet and test the ubiquity of planetary system coplanarity. The survey will detect planets in all orbital orientations, allowing unambiguous statements to be made about the existence or absence of planets around individual stars, unlike transit and RV surveys.

Table 2. SIM broad planet survey

\begin{tabular}{|l|c|}
\hline Number of target stars & 2100 \\
\hline \# Terrestrial planets $\left(0.3-10 \mathrm{M}_{\oplus}\right)$ & 84 \\
\hline \# Neptunes $\left(10-30 \mathrm{M}_{\oplus}\right)$ & 42 \\
\hline \# Jupiters $\left(30 \mathrm{M}_{\oplus}-10 \mathrm{M}_{\text {Jupiter }}\right)$ & 300 \\
\hline Total number of planets & 426 \\
\hline
\end{tabular}

the exoplanet population.
This survey is complementary to the GAIA planet survey. GAIA covers approximately $\sim 50,000$ targets but with a much lower $(\sim 70$ $\mu$ as) equivalent single-measurement precision. While the GAIA mission will detect Jovian planets, the SIM broad planet survey will detect planets $\sim 20$ times less massive.

The survey targets 2,100 stars (Table 2). The sample is split into several groups, each designed to answer specific questions about

The primary target group will provide detailed statistics on the planetary populations around the full range of mainsequence stars. Although exceptional sensitivity will be achieved for every target, particular emphasis will be placed on the very low mass, high mass, and active stars that are extremely difficult to search with other planet-detection techniques.

Other target classes include stars in a wide range of age and metallicity, stars with dust disks, white dwarfs, stars in multiple systems, and stars with known exoplanets. Since SIM is a pointing observatory, other interesting targets will be searched for low-mass planets as they are discovered. 
We emphasize that the broad survey will produce a complete planetary census of the 2,100 target stars down to the top of the terrestrial-mass regime. Planet formation simulations (Ida \& Lin $2004 \mathrm{ab}, 2005$; Benz et al. 2006) as well as the microlensing detection of a rocky exoplanet (Dominik et al. 2006) suggest that terrestrial-mass planets are far more common than gas giants.

\section{4c. SIM Young Star Survey}

SIM's Young Stars and Planets Key Project will contribute a census of gas giant planets orbiting 150-200 stars with ages from 1 Myr to 100 Myr. These discoveries will lead to greater understanding of the formation and dynamical evolution of gas giant planets. The groundbreaking aspect of this investigation is not its mass sensitivity but its unique ability to probe an important epoch in the planet formation process about which little is currently known.

Ground-based advances in detecting young Jupiter-mass planets are likely to come from IR coronagraphic imaging and extreme AO. Such techniques have, to date, produced a few intriguing objects at wide separations (20-100 AU) from very young (<10 Myr) host stars, e.g. 2M1207 (Chauvin et al. 2005) and GQ Lup (Neuhauser et al. 2005). These companions, potentially, have masses well into the planetary regime, depending on the choice of evolutionary models that predict the brightness of "young Jupiters" as a function of mass and age (Baraffe et al. 2002). Unfortunately, dynamical determinations of mass (which would distinguish planets from brown dwarfs) are impossible for companions on such distant orbits. Multiple fragmentation events (Boss 2001) rather than core accretion in a dense disk (Ida \& Lin 2004) may be responsible for the formation of these distant objects.

A Jupiter orbiting $\sim 1$ AU away from a $0.8 \mathrm{M}_{\odot}$ star at the distance of the youngest stellar associations (1-10 Myr), such as Taurus and Chamaeleon, would produce an astrometric amplitude of $8 \mu$ as, easily detected with SIM. At the 25-50 pc distances of the nearest young stars (10-50 Myr), such as members of the $\beta$ Pic and TW Hya groups, the same stellar system would have an enormous astrometric amplitude in excess of $100 \mu$ as.

In a survey of 200 young stars, SIM can expect upwards of 10-20 planetary systems (assuming that only the presently known fraction of stars, $5-10 \%$, have planets). The sensitivity threshold has been set to ensure the detection of Jupitermass planets in the critical orbital range of 1 to 5 AU. These observations, when combined with the results of planetary searches of mature stars, will allow us to test theories of planetary formation and early solar system evolution. By searching for planets around pre-main sequence stars carefully selected to span an age range from 1 to 100 Myr, we will learn at what epoch and with what frequency giant planets are found at the water-ice "snowline" where they are expected to form (Pollack et al. 1996). This will provide insight into the physical mechanisms by which planets form and migrate from their place of birth and about their survival rate.

These observations will provide data, for the first time, on such important questions as: What processes affect the formation and dynamical evolution of planets? When and where do planets form? What is the initial mass distribution of planetary systems around young stars? How might planets be destroyed? What is the origin of the eccentricity of planetary orbits? What is the origin of the apparent dearth of companion objects with masses between gas giant planets and brown dwarfs seen in mature stars? How might the formation and migration of gas giant planets affect the formation of terrestrial planets?

About half of the sample will be used to address the "where" and "when" of planet formation by observing classical T Tauri stars which have massive accretion disks as well as weak-lined T Tauri stars. The other half of the sample will be stars from $5 \mathrm{Myr}$ through $10 \mathrm{Myr}$ (thought to mark the end of prominent disks) and ending around the $100 \mathrm{Myr}$ age, to study the effects of dynamical evolution and planet destruction (Lin et al. 2000).

\section{SIM ASTROMETRY COMPLEMENTS OTHER EXOPLANET SEARCH AND CHARACTERIZATION TECHNIQUES}

Astrometry and radial velocity are complementary approaches to planet detection. RV is more sensitive to short period planets and astrometry more sensitive to long period planets. The SIM science team has members from the two leading RV planet search groups. Extensive RV data from current instruments have been studied to derive the sensitivity limits due to motion of the stellar atmospheres of the target stars. At $\sim 1 \mathrm{~m} / \mathrm{sec}$, it is temporally correlated stellar noise, caused 
by p-modes, supergranulation, meridional oscillations, and magnetic cycles. These stellar velocity fields have characteristic time scales of days to decades, causing temporally correlated Doppler shifts of $2 \mathrm{~m} / \mathrm{s}$ (for G stars) and 1 $\mathrm{m} / \mathrm{s}$ (for K stars). This $1 \mathrm{~m} / \mathrm{s}$ correlated noise floor prevents the Doppler method from securely detecting planets under $10 \mathrm{M}_{\oplus}$ beyond $0.5 \mathrm{AU}$ from the star.

Astrometry is the only technique that permits accurate measurement of both mass (a key parameter for planetary science) and orbit inclination. The RV technique has revealed 20 multi-planet systems, $13 \%$ of the total number of known exoplanets. However, we do not know if coplanar orbits are common or rare. SIM astrometry can answer this key question. SIM can also detect Saturn-mass planets in five year orbits around solar-like stars at 800 pc. Finding additional planets in large orbits around stars found by missions such as Kepler will tell us if our own solar system is common. The completeness of astrometry is important in that non-detection is significant.

The SIM astrometric search is also useful as a precursor to planning a direct detection campaign, such as with TPF. Terrestrial planets in the HZ that are found by SIM would become prime targets for a follow-up space mission to obtain spectra of the planets: both missions study samples of nearby solar-type stars. SIM astrometry also provides information about where and when to observe with TPF, i.e. avoiding TPF observations when the planet resides within the inner working angle. Non-detection by SIM is also important, helping to prioritize TPF target stars.

\section{REMAINING TECHNICAL RISKS}

The SIM technology development program was completed to TRL (Technical Readiness Level) 6 in June 2005 (Laskin 2006). It has been extensively reviewed and was formally signed off by NASA HQ in March 2006. The technology program has demonstrated the extreme performance $(0.6 \mu$ as narrow-angle single-measurement and $2.4 \mu$ as wide-angle mission accuracy) needed for exoplanet detection with substantial margins. Instrumental errors in the SIM testbed (chopped) integrate down as $1 / \sqrt{ } \mathrm{T}$ to a systematic error floor of less than 100 nano-arcsec.

SIM has completed virtually all of its Formulation Phase (A/B) work, including completion of technology development as described above. This has resulted in a mature design with well-understood cost and schedule (also verified by NASA external independent cost estimates).

What then are the remaining risks to the use of this technology (by SIM or another mission) for finding and characterizing planets?

The technology program has not only shown that it is possible to build all of the necessary components (hardware and software) needed for a $0.6 \mu$ as astrometry mission with a systematic error floor below $0.1 \mu$ as. It has further shown that these components work together, in a complete system, to achieve these results (Laskin 2006).

SIM's continued engineering development of these hardware components into robust flight qualifiable hardware and software has shown that, even for the most sensitive picometer-hardware, these components can be built using conventional flight hardware fabrication techniques with no degradation in performance from that of the technology demonstrations (Marr 2006, Marr 2007).

Technology testbeds developed for SIM have demonstrated all key algorithms for control and measurement of the instrument. The project has demonstrated a distributed computing environment that supports the strict timing requirements for high-bandwidth control of hardware that is distributed over a very large structure (for example, SIM or TPF). Processing of the SIM testbed measurements have taught the team how to process the instrument output to achieve the required measurement accuracy and precision in ways not anticipated early in the technology program, significantly relaxing hardware requirements. These data-processing lessons-learned should form the basis for ground processing for any flight interferometer.

Interactions between the spacecraft bus and the instrument are also well understood (for SIM). These interactions include: vibration suppression (simple two-stage passive vibration isolation is sufficient), attitude stabilization for beam walk suppression (using the two guide interferometers as a micro-arcsecond two-axis star tracker to control the spacecraft attitude control system; $\sim 10^{6}$ times more accurate than a typical spacecraft star tracker), and torque feed- 
forward from the instrument to the spacecraft attitude control system to minimize attitude disturbances resulting from the motion of instrument siderostats and delay lines.

With well over \$200M invested in the development of this technology, remaining risks for a user of this technology are the usual ones that occur during the implementation of any large system (manufacturing errors, interface mismatches, personnel errors, etc.).

\section{SUMMARY}

This paper describes the use of a space-based long-baseline optical Michelson stellar interferometer for detection of habitable planets around $\sim 130$ nearby stars and that will provide a census of planets around $\sim 2100$ stars to place our solar system in a broader context. SIM will open a new era of the characterization of rocky planets in the solar neighborhood. All key hardware and software elements, subsystems, and systems have been demonstrated at, or better than, the performance levels required to meet the necessary $1.0 \mu$ as precision. The SIM project's ongoing development of hardware assemblies into flight-like assemblies continues to show that standard flight hardware development processes are sufficient for building and testing these assemblies. Currently, there are no significant technical risks to the full-scale deployment of a space-based astrometry mission similar to the SIM mission. Further information about this technology program and about the SIM mission can be found on the SIM website (http://planetquest/SIM/sim_index.html).

Having completed its technology program in 2005 and almost completed Formulation Phase (Phase B), SIM is a lowrisk mission with a well-established cost that is ready for immediate full scale development. Humanity could discover its first $1 \mathrm{M}_{\oplus}$ exo-planet in the habitable zone of a nearby star in less than a decade!

\section{REFERENCES}

Bahcall, J.N. ,"The Decade of Discovery in Astronomy and Astrophysics, National Academies Press, ISBN 0-0309-04381-6, 1991

Baraffe, I. et al., A\&A, 382, 563, 2002

Benz, W. et al., Proceedings IAU Colloquium 200, 2006

Boss, A., ApJ, 551, L167, 2001

Catanzarite, J. et al, Astrometric detection of terrestrial planets in the habitable zone of nearby stars with SIMPlanetQuest, Publications of the Astronomical Society of the Pacific (PASP), September 2006

Chauvin, G. et al., A\&A, 438, L25, 2005

Cumming, A., et al., ApJ, in press, 2007

Dominik, M. et al., A\&G 47, 3, 25, 2006

Ida, S. \& Lin, D.N.C ApJ, 604, 388, 2004a

Ida, S. \& Lin, D.N.C ApJ 616, 567, 2004b

Ida, S. \& Lin, D.N.C ApJ 626, 1045, 2005

Laskin, R.A., Successful Completion of SIM-PlanetQuest Technology, SPIE Vol. 6268, 626823, 2006

Laskin, R.A., et al, SIM-PlanetQuest Technology Completion: A Retrospective View, IAC-06-A3.P.1.05, 2006

Lin, D.N.C. et al., Protostars and Planets IV, 1111, 2000

Marr, J. C. et al., ExoPTF White Paper, Technology for Astrometric Detection of Nearby Earth-Mass Habitable Zone Planets from Space, 2007

Marr, J.C. et al, SIM PlanetQuest - Progress Report, SPIE Vol. 6268, Symposium on Astronomical Telescopes, 2006

McElroy, J.H. \& Huchra, J.P., Letter to Dr. Edward Weiler in response to Dr. Weiler's March 18, 2002 letter to J.H. McElroy requesting that the NRC CAA provide an assessment of SIM, September 12, 2002.

McKee, C. \& Taylor, J., 2001 Astronomy and Astrophysics in the New Millennium, National Academies Press, ISBN-309-0731-2. 2001

Neuhäuser, R. et al, A\&A, 435, L13, 2005

Pollack, J., et al, Icarus 124, 62, 1996

Shao, M., et al, ExoPTF White Paper, SIM PlanetQuest - The Most Promising Near-Term Technique to Detect and Find Masses and 3-D Orbits of Nearby Habitable Planets, 2007

Unwin, S., et al, Taking the Measure of the Universe: Precision Astrometry with SIM PlanetQuest, 2007 Publications of the Astronomical Society of the Pacific (in press). 